\title{
Review
}

\section{Tramadol, Pharmacology, Side Effects, and Serotonin Syndrome: A Review}

Burton D. Beakley, MD', Adam M. Kaye, PharmD², and Alan D. Kaye, MD PhD ${ }^{1,3}$

From: ${ }^{2}$ Department of Anesthesiology, Tulane School of Medicine, New Orleans, LA; ${ }^{2}$ Thomas J. Long School of Pharmacy and Health Sciences University of the Pacific, Stockton, CA; 3 Department of Anesthesiology, Louisiana State School of Medicine, New Orleans, LA

Address Correspondence: Alan David Kaye, MD, PhD Professor and Chairman, Department of Anesthesiology Professor

Department of Pharmacology Louisiana State University School of Medicine 1542 Tulane Avenue, Room 656, New Orleans, LA 70112 E-mail: akaye@lsuhsc.edu

Disclaimers: P. 400

Manuscript received: 06-18-2015 Accepted for publication: 06-22-2015

Free full manuscript: www.painphysicianjournal.com
Background: Serotonin syndrome is a mild to potentially life-threatening syndrome associated with excessive serotonergic activity within the central nervous system. Serotonin syndrome is associated with medication use, drug interactions, and overdose. While serotonin syndrome is often associated with the use of selective serotonin inhibitors (SSRI), an increasing number of reports are being presented involving the use of tramadol.

Methods: This review article contains an overview of serotonin syndrome while specifically looking at tramadol's pharmacology and risk factors for serotonin syndrome. With tramadol's increasing popularity, the goal of this article is to make physicians more alert and aware of this potential side effect associated with tramadol.

Conclusions: In conclusion, with the increasing incidence of serotonin syndrome, prescribing physicians should be aware of and educate their patients on the potential side effects of tramadol. It is important that the prescribing physician reviews patient medications for concurrent serotonergic drugs and monitors for potential abuse.

Key words: Tramadol, serotonin syndrome, drug interactions, analgesics

Pain Physician 2015; 18:395-400 ramadol is a dual action analgesic agent utilized in the treatment of a variety of pain syndromes (1). In recent years, tramadol, an analogue of codeine, has gained popularity as an analgesic agent. Serotonin syndrome is associated with medication use, drug interactions, and overdose. While serotonin syndrome is often associated with the use of selective serotonin reuptake inhibitors (SSRIs), evolving literature has identified a very significant side of effect of tramadol: the development of this potentially catastrophic clinical syndrome. This review focuses on the pharmacology, drug interactions, and potential side effects of tramadol, including its role in the development of serotonin syndrome.

Data indicates that the incidence of serotonin syndrome is increasing with the widespread use of sero- tonergic drugs in practice (2-18). Serotonin syndrome has been reported in patients of all ages with a known risk factor being polypharmacy and severe cases often coinciding with drug interactions (19-24). As the number of case reports involving serotonin syndrome and tramadol increase, it is important to be aware of patients with increased risk for these complications.

\section{Presentation/Mechanism}

Serotonin syndrome is the result of excess serotonin at the 5-HT receptor, it is classically a druginduced disorder characterized by change in mental status, autonomic function, and neuromuscular activity (10). While classically patients present with a triad of symptoms, the presentation is highly variable and can range from benign to life-threatening clinical 
symptoms $(2,5,10)$. Serotonin syndrome has been reported in all age groups from newborns to the elderly (2). Even with the increase in serotonergic acting drugs, serotonin syndrome is under-reported secondary to incorrect diagnosis, mild symptoms, and physician unawareness $(2,3)$.

Serotonin syndrome is the consequence of overactive central and peripheral serotonin receptors, specifically the $5-\mathrm{HT} 1 \mathrm{~A}$ and $5-\mathrm{HT} 2 \mathrm{~A}$ receptors (5). Serotonin (5-hydroxytryptamine or 5-HT) is derived from the decarboxylation and hydroxylation of tryptophan. It is released into the synaptic cleft where it has been shown to bind at least 7 separate receptors modulating behavior, thermoregulation, and attention centrally, while peripherally influencing gastrointestinal motility, bronchoconstriction, vasoconstriction, and platelet aggregation (2). Of the 7 receptors serotonin binds, it is 5-HT1A and 5-HT2A that are most associated with serotonin syndrome (14). Serotonin is then degraded by monoamine oxidase (MAOA) to be excreted in the urine as 5-hydroxyindoleacetic acid.

Serotonin syndrome is associated with the use of serotonergic drugs. These include drugs that influence serotonin uptake, metabolism, synthesis, release, and serotonin receptor activity; also drugs with the ability to interfere with cytochrome P450 metabolism, specifically CYP2D6 and CYP3A4. Antidepressants (serotonin norepinephrine reuptake inhibitors [SNRI], SSRI, monoamine oxidase inhibitor [MAOI]), antipsychotics, analgesics, antiemetics, cough suppressants, and dietary supplements are drugs recognized to be involved in serotonin syndrome (Table 1). SSRIs are the most reported drugs associated with serotonin syndrome by the National Poison Data Center (12), and commonly it is the concurrent use of multiple serotonergic drugs that results in serotonin syndrome. However, serotonin syndrome is reported in patients with initiation of a single serotonin agent or recent dose change or overdose.

\section{Diagnosis}

Diagnosis is based on clinical findings, with no specific laboratory or radiographic testing for confirmation (2). A diagnosis by a medical toxicologist is the gold standard (5). However, in the clinical setting, a thorough history and physical/neurologic exam is essential. A patient's history will usually include symptoms developing within 24 hours of serotonergic medication addition/ initiation, dose change, or overdose. The patient should be asked to describe the onset and duration of symp- toms along with any co-morbidity (e.g., end stage renal disease) which could lead to worsening complications. Next a detailed list of the patient's prescription drugs, over-the-counter medications, illicit drug use, and dietary supplements should be obtained. The physician should pay particular attention for MAOI use and/or intentional intoxication as these patients often develop greater toxicity resulting in adverse outcomes (2).

Patients can display signs of mental status change including agitation, anxiety, disorientation, restlessness, and excitement. Neuromuscular abnormalities include tremors, clonus, hyperreflexia, muscle rigidity, bilateral Babinski sign, and akisthesia, and autonomic hyperactivity presenting with hypertension, tachycardia, tachypnea, hyperthermia, vomiting, diarrhea, arrhythmias, and shivering (Table 2). Patients with mild symptoms commonly present with mild hypertension and tachycardia, mydriasis, diaphoresis, shivering, tremor, myoclonus, and hyperreflexia; with no hyperthermia. Patients with moderate symptoms will begin to develop hyperthermia up to 40 degrees Celsius, hyperactive bowel sounds, agitation, pressured speech, and horizontal ocular clonus. Severe cases can expect hyperthermia greater than 41 degrees Celsius, vital sign instability, delirium, and muscle rigidity (5). Complications from severe cases can include seizure, rhabdomyolysis, arrhythmias, and respiratory arrest.

Diagnostic criteria have been developed to assist the physician in diagnosing serotonin syndrome. Hunter Serotonin Toxicity Criteria (HSTC) has replaced the originally used Sternbach Criteria due to its increased sensitivity and specificity. HSTC has been shown to have a sensitivity of $84 \%$ and specificity of $97 \%$ in the diagnosis of serotonin syndrome when compared to the gold standard of a medical toxicologist (4). The HSTC requires a patient to have consumed a serotonergic drug and have one of 5 conditions (Table 3 ).

\section{Management}

Management begins with quick recognition and diagnosis to avoid the morbidity and mortality associated with serotonin syndrome. Once serotonin syndrome is established, treatment is centered on five principles (Table 4); the mainstay of therapy being prompt discontinuation of all offending medications. Symptomatic improvement is usually seen within the first 24 hours after discontinuation of serotonergic drugs. Treatment is otherwise supportive management of symptoms, commonly including agitation, hyperthermia, and autonomic dysfunction. This includes supportive care for 
Table 1. Drugs commonly implicated in inducing serotonin syndrome by mechanism.

Inhibitors of Serotonin Reuptake

Selective serotonin reuptake inhibitors (SSRIs): Fluoxetine, Paroxetine, Sertraline, Citalopram, Fluvoxamine

Serotonin-norepinephrine reuptake inhibitors (SNRIs): Venlafaxine, Desvenlafaxine, Duloxetine, milnacipran

Tricyclic antidepressants (TCAs): Amitriptyline, Nortriptyline, Protriptyline Clomipramine, Imipramine, Desipramine, Trimipramine,

Amoxapine, Doxepin, Maprotiline

Tricyclic skeletal muscle relaxant: Cyclobenzaprine

Dopamine-norepinephrine reuptake inhibitors: Buproprion

Serotonin modulators: Trazadone, Nefazadone

Triazolopyridine antidepressant: Trazodone

Tetracyclic antidepressant: Maprotiline, Mirtazapine

Phenylpiperidine opioids: Fentanyl, Dextromethorphan

5-HT3 receptor antagonists: Ondansetron, Granisetron

Local anesthetics: Cocaine

Herbal supplements: St. John's Wort (Hypericum perforatum)

Tramadol

Meperidine

Methadone

MDMA (Ecstasy)

Inhibitors of Serotonin Metabolism

Monoamine oxidase inhibitors (MAOIs): St. John's Wort, Linezolid, Methylene Blue, Tranylcypromine, Selegiline, Phenelzine, Isocarboxacid,

Furazolidone, Syrian Rue,

Selective MAO-B inhibitor: Rasagiline

Increase Serotonin Synthesis

L-tryptophan

Increase Serotonin Release

Amphetamines and amphetamine derivatives: Methamphetamine, Fenfluramine, Phentermine

Dopamine agonists: L-dopa and Bromocryptine

MDMA (Ecstasy)

Ethanol

Cocaine

Lithium

Serotonin Receptor Agonism

Antidepressants: Buspirone, Trazadone, Mirtazapine

Antimigraines (Triptans) Almotriptan, Eletriptan, Frovatriptan, Naratriptan, Rizatriptan,Sumatriptan, Zolmitriptan

Anticonvulsants (antiepileptic drugs) : Valproic Acid, Carbamazepine

Ergot alkaloid derivatives: Methylergonovine, Ergotamine

Fentanyl

Metoclopramide

Buspirone

Lysergic acid diethylamide (LSD)

Increases Sensitivity of Postsynaptic Receptor

Lithium

Modified from "Pharmacology Considerations in Serotonin Syndrome," Gayle J, Volpi J, Kaye AD, Kaye AM, Essentials of Pharmacology with Black Box Warnings, editors AD Kaye, AM Kaye, and R Urman, Springer, 2014, chapter 49, pages 797 - 810, with permission.

vital instability with continuous cardiac monitoring, intravenous fluids, and oxygen therapy to maintain saturations greater than $93 \%$. For agitation, administration of benzodiazepines is preferred over physical restraints. In the moderately to severely ill, administration of serotonin antagonists should be considered. After symptomatic improvement the patient needs to be evaluated for risk/benefits of continued serotonergic drugs.

\section{Tramadol}

Tramadol (( \pm )cis-2-[(dimethylamino)methyl]-1-(3methoxyphenyl)-cyclohexanol hydrochloride) is growing in popularity as physicians look into alternative analgesics hoping to avoid the side effects of addiction, respiratory depression, abuse, etc. While tramadol is recognized to have less potential for abuse and respiratory depression, it is not without its risk. 
Table 2. Symptoms of serotonin syndrome.

\begin{tabular}{|c|c|}
\hline Symptom Cluster & Symptoms \\
\hline \multirow{5}{*}{ Altered Mental Status } & Agitation \\
\hline & Anxiety \\
\hline & Disorientation \\
\hline & Restlessness \\
\hline & Excitement \\
\hline \multirow{6}{*}{ Neuromuscular Abnormalities } & Tremor \\
\hline & Clonus \\
\hline & Hyperreflexia \\
\hline & Muscle Rigidity \\
\hline & Bilateral Babinski Sign \\
\hline & Akisthesia \\
\hline \multirow{8}{*}{ Autonomic Hyperactivity } & Hypertension \\
\hline & Tachycardia \\
\hline & Tachypnea \\
\hline & Hyperthermia \\
\hline & Vomiting \\
\hline & Diarrhea \\
\hline & Arrhythmias \\
\hline & Shivering \\
\hline
\end{tabular}

From Serotonin Syndrome (5), with permission

Table 3. Classic Hunter serotonin toxicity criteria: decision rules.

In the presence of a serotonergic agent PLUS one of the following groups:

- Spontaneous clonus

- Inducible clonus with agitation or diaphoresis

- Ocular clonus with agitation or diaphoresis

- Tremor and hyperreflexia

- Hypertonia, temperature above $100.4\left(38^{\circ} \mathrm{C}\right)$, and ocular or inducible clonus

Table 4. Five basic principles central to management of serotonin syndrome.

- Discontinue all serotonergic agents

- Maintaining vital sign stability with supportive management

- Benzodiazepines for sedation

- When appropriate administration of serotonin antagonists

- Careful assessment of risk/benefits of continuing serotonergic agents after resolution of symptoms

Modified from Boyer EW, Traub SJ, Grayzel J. Serotonin Syndrome. In: UpToDate.
Table 5. Affinity of tramadol, two enantiomers and active metabolite.

\begin{tabular}{|c|c|c|c|}
\hline \multirow{2}{*}{$\begin{array}{c}\text { Opioid } \\
\text { Component }\end{array}$} & $\begin{array}{c}\text { Affinity } \\
\text { for Opioid } \\
\text { Receptors }\end{array}$ & \multicolumn{2}{|c|}{ Reuptake Inhibition } \\
\cline { 2 - 4 } & $\boldsymbol{\mu}$ & Norepinephrine & Serotonin \\
\hline$( \pm)$ Tramadol & ++ & + & + \\
\hline$(+)$ Tramadol & +++ & + & ++ \\
\hline$(-)$ Tramadol & + & ++ & + \\
\hline$(+)$ M1 & ++++ & & \\
\hline Morphine & +++++ & & \\
\hline
\end{tabular}

Modified from Benzon H. Practical Management of Pain. 5th ed. Mosby, St. Louis, 2014.

Tramadol has a unique dual action of pain relief, acting both as a central opiate agonist and central nervous system (CNS) reuptake inhibitor of norepinephrine and serotonin. Tramadol exist as 2 enantiomers with analgesic properties, both with different mechanisms of action. (+)-Tramadol and it metabolite O-desmethyltramadol (M1) act as selective mu-receptor agonists altering the release of nociceptive neurotransmitters (1). The mu activity of tramadol is around 10 fold less than that of codeine with the M1 metabolite having 300 times more affinity for mu receptor compared to its parent compound (7) (Table 5). Also (+)-tramadol inhibits serotonin reuptake and (-)-tramadol inhibits norepinephrine reuptake $(1,15)$; the reuptake inhibition of serotonin and norepinephrine enhance the inhibitory descending pathways associated with pain transmission in the CNS (1).

Tramadol is administered orally, rectally, sustained release, and in solution for IV/IM administration. Oral administration of tramadol has rapid absorption and distribution with maximum serum concentration reached after 2 hours (7). Tramadol is capable of passing the placental barrier and small amounts of tramadol and its metabolite have been detected in breast milk (1).

Tramadol undergoes extensive first pass metabolism within the liver by $\mathrm{O}$ - and $\mathrm{N}$ - demethylation and conjugation reactions. The kidneys are responsible for $90 \%$ excretion of tramadol and its metabolites; the remaining $10 \%$ is excreted through feces (1). The demethylation reaction is catalyzed by enzyme cytochrome $\mathrm{P} 450$ CYP2D6 and CYP3A4/CYP2B6 to metabolites O- and $\mathrm{N}$-demethylated tramadol respectively $(1,15)$. Of the 23 metabolites that have been identified M1, M2, and M5 are the main metabolites and metabolite O-desmethyl- 
tramadol (M1) is the only metabolite with analgesic properties $(1,7)$. The half-life $(\mathrm{t} 1 / 2)$ of tramadol is $5-6$ hours and 8 hours for the M1 metabolite (7). Because of its hepatic metabolism and renal clearance, impairment in these systems may require dose modifications.

Tramadol is currently recommended for the treatment of moderate to moderately severe pain in patients unresponsive to previous oral therapies or who have a contraindication to selective cox 2 inhibitors or nonselective cox inhibitors (7). Tramadol has shown to have a lower risk for addiction with chronic use when compared with other opiates. Most common side effects include nausea, vomiting, sweating, fatigue, sedation, and dry mouth (7). More severe side effects include angioedema, increased effect of anticoagulants, and serotonin toxicity (7).

\section{Tramadol and Serotonin Syndrome}

While SSRIs are the most commonly reported drug associated with serotonin syndrome, physicians should be aware of tramadol as a potential single agent cause for serotonin syndrome. Tramadol is known to inhibit serotonin reuptake and may induce serotonin release at high doses (23). On case review, tramadol induced serotonin syndrome was often associated with unintentional/intentional overdose of the drug $(8,16,17)$. A retrospective review found acute ingestions represented $90 \%$ of tramadol exposure reported to the poison control system, with the main cause being attempted suicide (8). Tramadol overdose symptoms included CNS depression, nausea and vomiting, tachycardia, and seizure $(8,9)$.

While overdose is a common cause for tramadol's side effects, as a single agent there is also the concern for patients with impaired metabolism of tramadol and/or deficient serotonin uptake. Poor metabolizers of tramadol are at risk for elevated (+)-tramadol levels (1). It is (+)-tramadol that is associated with the serotonergic reuptake inhibition activity (1). As noted in "Clinical Pharmacology of Tramadol" (1), polymorphisms of cytochrome P450 CYP2D6 resulted in varying degrees of metabolism to active metabolite M1 O-desmethyltramadol. Poor metabolizers resulted in low plasma levels of active $M 1$ resulting in inadequate pain control and concern for increased (+)-tramadol levels associated with serotonin reuptake inhibition $(1,13)$. Fox et al (6)looked at mice with serotonin transporter (SERT) deficiencies, a protein responsible for the termination of serotonin action, and found that tramadol alone can induce serotonin-like syndrome in rodents lacking one or 2 copies of serotonin transporter. Also mice lacking genes for monoamine oxidase (MAO) A and $B$, the enzyme responsible for breakdown of serotonin, showed increased serotonin levels and serotonin syndrome like behaviors when administered tramadol (18). Both these rodent models showed potential increased vulnerability in individuals with SERT and/or MAO polymorphisms $(6,18)$. These findings suggest a genetic susceptibility to developing serotonin syndrome influenced by the patient's ability to produce different ratios of $(+)$ and (-) tramadol enantiomers (13).

Another cause for concern is tramadol and the concurrent use of other serotonergic drugs including SSRIs, SNRIs, tricyclic antidepressants, MAOls, etc. Shatin et al (24), in a retrospective analysis, found $20.7 \%$ of people who received a tramadol prescription also received an antidepressant within 30 days. SSRIs inhibit CYP2D6 decreasing the analgesic activity of tramadol. The concurrent use of SSRIs with tramadol has been shown to induce serotonin syndrome through synergistic serotonergic action, along with the inhibition of CYP2D6, resulting in higher levels of $(+)$ tramadol enantiomer associated with serotonergic activity (1). Serotonin syndrome has been reported with the use of CYP2D6 inhibitors (ex. paroxetine, venlafaxine) and tramadol $(20,22)$.

\section{Conclusions}

Serotonin syndrome is a potentially life-threatening complication with a favorable prognosis if caught early. With the increasing number of serotonergic drugs and patients being treated with polypharmacy, physicians must be aware of the potential side effect profiles and interactions with these medications. With the most common incidence of serotonin syndrome with tramadol being due to concurrent serotonergic therapy, it will be important for physicians to weigh the risk/benefits of tramadol for pain management in this group. And while tramadol shows less risk for addiction, the risk of serotonin syndrome from tramadol overdose requires the physician to carefully monitor and follow patients while considering alternative therapies in patients with a history overdose. Lastly a basic understanding of tramadol's metabolism and excretion will help the physician avoid patient populations who could be more prone to developing serotonin syndrome.

\section{Disclaimer:}

There was no external funding in the preparation of this manuscript. 


\section{Conflict of interest}

Each author certifies that he or she, or a member of his or her immediate family, has no commercial association (i.e., consultancies, stock ownership, eq- uity interest, patent/licensing arrangements, etc.) that might pose a conflict of interest in connection with the submitted manuscript.

\section{References}

1. Grond S, Sablotzki A. Clinical pharmacology of tramadol. Clinical Pharmacokinetics 2004; 43:879-923.

2. Boyer EW, Traub SJ, Grayzel J. Serotonin Syndrome. In: UpToDate. 2015. Accessed 20 May 2015.

3. Boyer EW, Shannon M. The serotonin syndrome. N Engl ] Med 2005; 352:1112.

4. Dunkley EJ, Isbister GK, Sibbritt D, Dawson AH, Whyte IM. The Hunter Serotonin Toxicity Criteria: Simple and accurate diagnostic decision rules for serotonin toxicity. QJM 2003; 96:635-642.

5. Volpi-Abadie J, Kaye AM, Kaye AD.. Serotonin syndrome. The Ochsner Journal 2013; 13:533-540.

6. Fox MA, Jensen $C L$, Gallager PS, Murphy DL. Receptor mediation of exaggerated responses to serotonin-enhancing drugs in serotonin transporter (SERT)deficient mice. Neuropharmacology 2007; 53:643-656.

7. Benzon H. Practical Management of Pain. 5th ed. Mosby, St. Louis, 2014.

8. Marquardt KA, Alsop JA, Albertson TE. Tramadol exposures reported to statewide posion control system. Ann Pharmacotherapy 2005; 39:1039-1044.

9. Tashakori A, Afshari R. Tramadol overdose as a cause of serotonin syndrome: A case review. Clinical Toxicology 2010; 48:337-341.
10. Ferri F. Ferri's Clinical Advisor 2015. Mosby, St. Louis, 2014.

11. Igbal MM, Basil MJ, Kaplan J, Igbal MT. Overview of serotonin syndrome. Ann Clinical Psychiatry 2012; 24:310-318.

12. Bronstein AC, Spyker DA, Cantilena LR Jr., Rumack BH, Dart RC. 2011 Annual report of the American Association of Poison Control Centers' National Poison Data System (NPDS): 29th Annual Report. Clin Toxicol (Phila) 2012; 50:911.

13. Park S, Wackernah R, Stimmel G. Serotonin syndrome: Is it a reason to avoid the use of tramadol with antidepressants. Journal of Pharmacy Practice 2014; 27:71-78.

14. Mills KC. Serotonin syndrome. A clinical update. Crit Care Clin 1997; 13:763-783.

15. Paar WD, Frankus P, Dengler HJ. The metabolism of tramadol by human liver microsomes. Clin Investig 1992; 70:708-710.

16. Takeshita J, Litzinger M. Serotonin syndrome associated with tramadol. Prim Care Companion J Clin Psychiatry 2009; 11:273.

17. Tashakori A, Afshari R. Tramadol overdose as a cause of serotonin syndrome: A case series. Clin Toxicol (Phila) 2010; 48:337-341.

18. Fox MA, Panessiti MG, Moya PR, Tolliver TJ, Chen K, Shih JC, Murphy DL. Mu- tations in monoamine oxidase (MAO) genes in mice lead to hypersensitivity to serotonin-enhancing drugs: Implications for drug side effects in humans. Pharmacogenomics Journal 2013; 13:551-557.

19. Peacock LE, Wright R. Serotonin syndrome secondary to tramadol and citalopram. Age Ageing 2011; 40:528.

20. Houlihan DJ. Serotonin syndrome resulting from coadministration of tramadol, venlafaxine, and mirtazapine. Ann Pharmacotherapy 2004; 38:411-413.

21. Mahlberg R, Kunz D, Sasse J, Kirchheiner J. Serotonin syndrome with tramadol and citalopram. Am J Psychiatry 2004; 161:1129.

22. Lantz MS, Buchalter EN, Giambanco V. Serotonin syndrome following the administration of tramadol with paroxetine therapy. Int Clin Psychopharmacol 1997; 12:181-182.

23. Reimann W, Schneider F. Induction of 5-hydroxytryptamine release by tramadol, fenfluramine, and reserpine. Eur J Pharmacol 1998; 349:199-203.

24. Shatin D, Gardner JS, Stergachis A, Blough D, Graham D. Impact of mailed warning to prescribers on the co-prescription of tramadol and antidepressants. Pharmacoepidemiol Drug Saf 2005; 14:149-154. 